Conclusion Estimates of stress MBF and MPR by perfusion-CMR in this study were greater in diastole than systole in normal and $\mathrm{CAD}$ patients. Although the diagnostic accuracy of both phases was similar, the MPR cut-off values were different. These observations are relevant to any form of dynamic myocardial perfusion assessment and are of particular importance to promising developments in $3 \mathrm{D}$ perfusion-CMR and CT perfusion imaging where the acquisition phase may be specifically chosen. Different estimates of MBF and different MPR cut-off values between phases mean a universal standard needs to be agreed for $3 \mathrm{D}$ acquisitions.

\section{ASSESSMENT OF ISCHAEMIC BURDEN IN PATIENTS WITH THREE-VESSEL CORONARY ARTERY DISEASE USING HIGH- RESOLUTION MYOCARDIAL PERFUSION CARDIOVASCULAR MRI}

doi:10.1136/heartjnl-2012-301877b.86

${ }^{1} \mathrm{M}$ Motwani, ${ }^{1} \mathrm{~T}$ A Fairbairn, ${ }^{1} \mathrm{~N}$ Maredia, ${ }^{2} \mathrm{~S}$ Kozerke, ${ }^{1} \mathrm{~J} \mathrm{P}$ Greenwood, ${ }^{1} \mathrm{~S}$ Plein. ${ }^{1}$ University of Leeds, Leeds, UK; ${ }^{2}$ University and ETH Zurich, Zurich, Switzerland

Introduction Patients with three-vessel disease (3VD) have a worse prognosis than those with less extensive disease; but detecting a 3VD pattern of ischaemia can be very challenging due to balanced hypoperfusion. However, a large ischaemic burden can also correctly stratify patients with $3 \mathrm{VD}$ as high-risk and prompt the appropriate management. We hypothesised that high-resolution perfusion-CMR would detect more ischaemic burden than standard-resolution due to better detection of subendocardial ischaemia. This study compared ischaemic burden detected by standard-resolution and high-resolution cardiovascular magnetic resonance (CMR) perfusion imaging in patients with 3VD.

Methods CAD was defined as coronary stenosis $>70 \%$ (QCA). 48 patients (24 with 3VD; 24 with no CAD) underwent stress/rest perfusion-CMR (1.5T Philips) with standard-resolution $(2.5 \mathrm{~mm}$ inplane) using twofold SENSE and on a separate visit high-resolution (1.6 $\mathrm{mm}$ in-plane) achieved by eightfold $\mathrm{k}$-t broad linear speed up technique (BLAST) acceleration (Abstract 086 figure 1). Perfusion was visually graded in each segment on a 5-point scale and summed to produce a perfusion score for each patient.

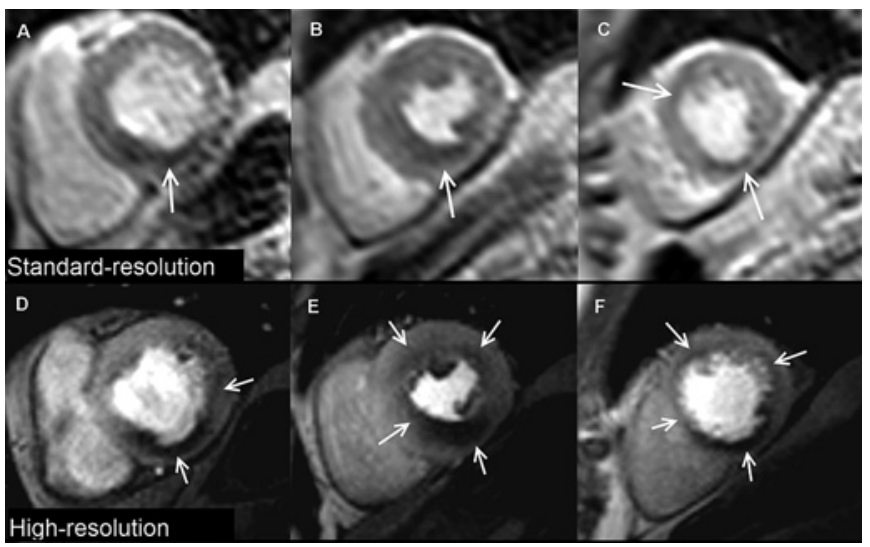

Abstract 086 Figure 1 Case example-standard-resolution shows perfusion defects (white arrows) in the basal inferior $(A)$, mid inferior, mid inferoseptal (B), apical anterior and apical inferior segments (C), High-resolution demonstrates additional ischaemia in the basal lateral (D), mid anterior and mid anterolateral segments (E) with a circumferential defect in the apical slice (F), perfusion defects are also better delineated at high-resolution and the transmural extent of ischaemia more clearly seen.
Results In the 3VD group, high-resolution identified more abnormal segments per patient $(7.3 \pm 3.7$ vs $5.2 \pm 3.9 ; p=0.01)$, more abnormal territories per patient $(2.0 \pm 0.9$ vs $1.46 \pm 1.0 ; p=0.02)$ and a higher overall perfusion score $(17.7 \pm 8.6$ vs $13.9 \pm 10.2 ; p=0.03)$. The number of segments with subendocardial ischaemia was greater for high-resolution (134 vs 70 segments; $47 \%$ vs $24 \%$; $p<0.001$ ) (Abstract 086 figure 2). The sensitivity, specificity and area under the curve (AUC) for identifying any perfusion defect were similar for both methods (high-resolution: $92 \%, 74 \%$ and 0.94 respectively vs standard-resolution: $79 \%, 84 \%$ and 0.87 ; $p>0.05$ ).

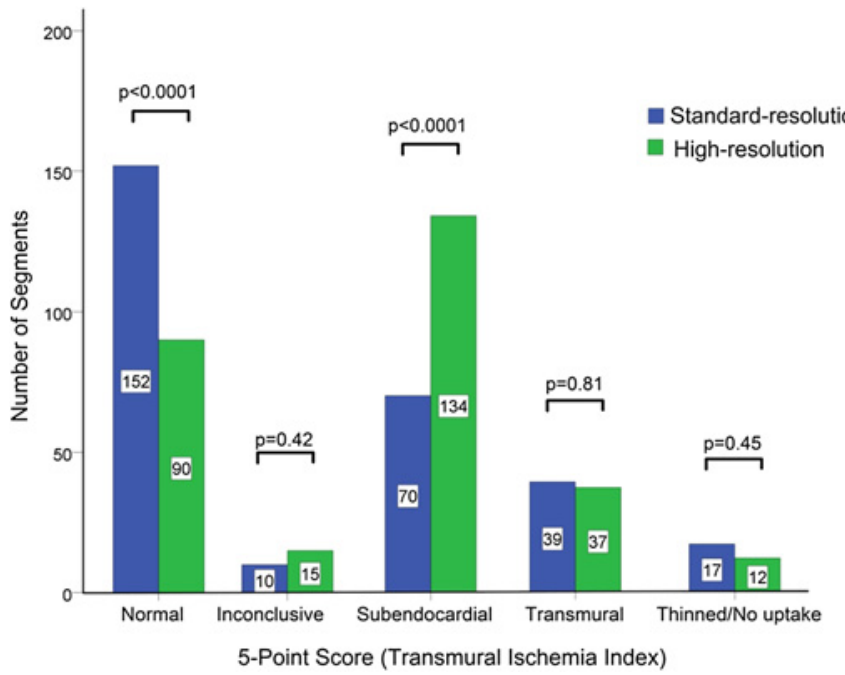

Abstract 086 Figure 2 Distribution of transmural ischaemia index. High-resolution perfusion CMR detected significantly more subendocardial ischaemia and fewer normal segments than standard-resolution in angiographically underperfused segments.

Conclusion In patients with $3 \mathrm{VD}$, high-resolution perfusion-CMR detected more ischaemic burden than standard-resolution by identifying more segments with subendocardial ischaemia. High-resolution perfusion-CMR therefore has incremental value in correctly stratifying this high-risk patient group.

\section{INCREMENTAL VALUE OF HIGH-RESOLUTION CARDIOVASCULAR MAGNETIC RESONANCE MYOCARDIAL PERFUSION IMAGING IN SUSPECTED CORONARY ARTERY DISEASE}

doi:10.1136/heartjnl-2012-301877b.87

${ }^{1} \mathrm{M}$ Motwani, ${ }^{1} \mathrm{~N}$ Maredia, ${ }^{1} \mathrm{~T}$ A Fairbairn, ${ }^{2} \mathrm{~S}$ Kozerke, ${ }^{1} \mathrm{~A}$ Radjenovic, ${ }^{1} \mathrm{~J}$ P Greenwood, ${ }^{1} \mathrm{~S}$ Plein. ${ }^{1}$ University of Leeds, Leeds, UK; ${ }^{2}$ University and ETH Zurich, Zurich, Switzerland

Introduction Although accelerated high-spatial-resolution cardiovascular magnetic resonance (CMR) perfusion imaging has recently been shown to be clinically feasible, there has not yet been a direct comparison with standard-resolution methods. We hypothesised that higher spatial resolution detects more subendocardial ischaemia and leads to greater diagnostic accuracy for the detection of angiographically defined $\mathrm{CAD}$. This study compared the diagnostic accuracy of high-resolution and standard-resolution CMR perfusion imaging in patients with suspected coronary artery disease (CAD). Methods A total of 111 patients with suspected CAD were prospectively recruited. All patients underwent two separate perfusion CMR studies on a 1.5 Tesla CMR scanner (Intera CV, Philips Healthcare, Best, the Netherlands), one with standard-resolution $(2.5 \times 2.5 \mathrm{~mm}$ in-plane resolution) and one with high-resolution $(1.6 \times 1.6 \mathrm{~mm}$ in-plane resolution) acquisition. High- 